\title{
Píldora anticonceptiva de emergencia: características de la demanda en una organización no gubernamental en Chile
}

\author{
Verónica Schiappacasse F. ${ }^{1,2 a}$, Tatiana Bascuñan C. ${ }^{2 a}$, Katherine Frez Z. ${ }^{2 a}$, \\ Isis Cortés H. ${ }^{2 b}$ \\ ${ }^{1}$ Escuela de Obstetricia y Puericultura, Facultad de Medicina, Universidad de Chile. ${ }^{2}$ Instituto Chileno de Medicina \\ Reproductiva (ICMER).
}

a Matrona. ${ }^{b}$ Psicóloga

\section{RESUMEN}

Antecedentes: En Chile, existe escasa información sobre las características de las usuarias de la píldora de emergencia y aún hay barreras para obtenerla, pese a existir una ley que garantiza su acceso. Objetivos: Indagar sobre el perfil de las mujeres que demandan la píldora de emergencia y las barreras para acceder al método en centros públicos de salud. Método: Estudio descriptivo y transversal. Se revisaron las fichas clínicas de usuarias de la píldora de emergencia atendidas en Prosalud Chile de enero de 2012 a marzo de 2013, para analizar edad, razón de uso, uso previo y antecedentes de no acceso. Resultados: Se analizaron 520 fichas clínicas. Edad promedio de las usuarias $21,8 \pm 6,8$ años. $50 \%$ usó el anticonceptivo porque tuvo un coito sin protección anticonceptiva y la otra mitad porque su método falló. El 71,7\% no había usado la píldora previamente y $0,8 \%$ (3 mujeres) la habían usado 3 y 4 veces; 8,8\% no obtuvo el anticonceptivo en centros públicos de salud. Conclusiones: La mayoría de las usuarias de la píldora de emergencia son jóvenes y la solicitan porque no usaron un anticonceptivo o éste les falló. El uso previo y frecuente es muy bajo. Aún existen barreras en el acceso en centros públicos de salud.

\section{PALABRAS CLAVE: Píldora anticonceptiva de emergencia, razones de uso, uso repetido, acceso.}

\section{SUMMARY}

Background: In Chile, the information about the characteristics of the users of emergency contraception pill is limited. In addition, some studies have revealed barriers to access to this method, despite the law guarantees its provision. Objectives: To enquire the profile of emergency contraceptive pills users and the barriers to its access in public health services. Methods: Descriptive and cross-sectional study. Clinical forms of users who requested the emergency contraceptive pill in Prosalud Chile from January 2012 to March 2013 were revised, in order to analyze age, reason to use, previous use and non-access history in public health services. Results: 520 clinical forms were analyzed. Users mean age was $21.8 \pm 6.8$ years. $50 \%$ of women demanded the pill because they did not use any contraceptive and another half because the contraceptive failed. $71.7 \%$ had never used the pill previously and only $0.8 \%$ ( 3 women) used it three and four times before. $8,8 \%$ could not obtain the pill in public health services. Conclusions: The majority of the emergency contraceptive pills users are young and they use it because they did not use a contraceptive or the contraceptive failed. The previous and frequent use is low. There are still barriers to access to emergency contraceptive pills in public health services.

KEY WORDS: Emergency contraceptive pills, reasons for use, patterns of repetitive use, access 


\section{INTRODUCCIÓN}

La píldora anticonceptiva de emergencia (PAE) es un método que se puede utilizar para prevenir un embarazo no planeado hasta cinco días después de una relación sexual no protegida. La PAE está indicada cuando no se usó un anticonceptivo, cuando éste falló o su uso fue incorrecto, y en los casos de violación sexual $(1,2,3)$.

El amplio y rápido acceso a la PAE favorece su uso (4) y disminuye la incidencia de embarazos no planeados, de abortos y sus consecuencias (5). La PAE permite que las mujeres ejerzan el derecho a controlar su fertilidad y regular el número de hijos e hijas que se desean tener y cuando hacerlo (6).

En 2001, el Instituto de Salud Pública aprobó por primera vez el registro de un producto de PAE. En 2004, la PAE fue incluida en la Norma de Violencia Sexual (7) y en 2006, en la Norma sobre Regulación de la Fertilidad del Ministerio de Salud (8). En 2005, el Ministerio de Salud, agregó la PAE de levonorgestrel al listado de medicamentos esenciales (9).

La introducción de la PAE generó un gran debate público y diversas demandas judiciales para prohibir su distribución y uso en el país. En 2008, el Tribunal Constitucional prohibió su entrega en la red de salud pública, pero no restringió su venta en farmacias. En 2010, se promulgó la Ley 20.418, que estableció la obligación de proveer la PAE a todas las mujeres, incluyendo a menores de 14 años, con la condición de informar a un adulto después de proveerla (10).

Chile permite la venta de la PAE sólo con receta médica retenida, lo que dificulta y retrasa su acceso (10). Algunos estudios han mostrado que aún existen barreras para acceder a la PAE, pese a existir un marco jurídico que asegura su acceso $(11,12)$.

El objetivo de esta comunicación es indagar sobre el perfil de las mujeres que demandan la píldora de emergencia y las barreras para acceder al método en centros públicos de salud.

\section{SUJETOS Y MÉTODO}

Se realizó un estudio cuantitativo y descriptivo, bajo un diseño transversal no experimental. Se revisaron las fichas clínicas de todas las usuarias que solicitaron la píldora anticonceptiva de emergencia desde enero de 2012 hasta marzo de 2013 en Prosalud Chile fundación chilena privada sin fines de lucro, miembro de la red de Prosalud Interamericana (PSIA), cuya misión es contribuir al ejercicio de una sexualidad responsable, sana y libre de riesgos, mediante diversas modalidades educativas. Se analizó la edad, razón de uso, uso previo y antecedentes de no acceso en centros públicos de salud. Las fichas fueron revisadas por una matrona del estudio. Los datos fueron ingresados a una base de datos utilizando el programa estadístico SPSS por una de las investigadoras del estudio. A todas las usuarias que se atendieron en Prosalud se les explicó que los datos de su ficha clínica serían utilizados en forma anónima y confidencial en una investigación, siempre y cuando ella lo autorizara. Todas las usuarias firmaron un consentimiento informado, en el cual se les explicó que su participación en el estudio era voluntaria, anónima y confidencial. Además, se solicitó la firma del consentimiento informado, del cual una copia quedó en poder de Fundación Prosalud y la otra con la usuaria. Los resultados finales del estudio fueron tabulados y analizados mediante el programa estadístico SPSS.

\section{RESULTADOS}

Durante el periodo analizado se atendieron 2.030 consultas por PAE, de estas, $520(25,6 \%)$ requirieron el medicamento y $1.510(74,4 \%)$ no necesitó la prescripción de este anticonceptivo porque no estaba en una situación de riesgo de embarazo (Tabla I). El número de consultas por PAE y el número de prescripciones de PAE realizadas en Prosalud Chile equivalen al $46,4 \%$ y al $45 \%$, del total de consultas de PAE y de entrega de PAE en el Sistema Público de Salud en 2010, respectivamente (13) (Tabla II). De las 520 usuarias a las que se les prescribió la PAE, $8(1,5 \%)$ tenían entre 11 a 14 años, $28,1 \% 15$ a 18 años, 29,6\% 19 a 22, 17,1\% 23 a 26 y el $23,7 \%$ tenía 27 y más años (Tabla III). El promedio de edad fue de $21,8 \pm 6,8$ años (rango: 13 a 50 años) (Tabla IV).

\section{Tabla I \\ CONSULTAS Y PRESCRIPCIONES DE LA PÍLDORA ANTICONCEPTIVA DE EMERGENCIA}

\begin{tabular}{lcc}
\hline Consultas & $\mathrm{n}$ & $\%$ \\
\hline Sin prescripción & 1.510 & 74,4 \\
Con prescripción & 520 & 25,6 \\
Total & 2.030 & 100 \\
\hline
\end{tabular}


Tabla II

CONSULTAS POR LA PÍLDORA ANTICONCEPTIVA DE EMERGENCIA SEGÚN ORGANIZACIÓN

\begin{tabular}{lccc}
\hline Consultas & $\begin{array}{c}\text { SNSS }^{*} \\
(2010)(n)\end{array}$ & $\begin{array}{c}\text { Prosalud } \\
(2012)(n)\end{array}$ & $\%$ \\
\hline Sin entrega de PAE & 3.218 & 1.510 & 46,9 \\
Con entrega de PAE & 1.156 & 520 & 45,0 \\
Total consultas de PAE & 4.374 & 2.030 & 46,4
\end{tabular}

*Sistema Nacional de Servicios de Salud. PAE: píldora anticonceptiva de emergencia.

Tabla III

EDAD DE LAS MUJERES QUE USARON LA PÍLDORA ANTICONCEPTIVA DE EMERGENCIA

\begin{tabular}{lcc}
\hline Rango edad (años) & $\mathrm{n}$ & $\%$ \\
\hline $11-14$ & 8 & 1,5 \\
$15-18$ & 146 & 28,1 \\
$19-22$ & 154 & 29,6 \\
$23-26$ & 89 & 17,1 \\
$\geq 27$ & 123 & 23,7 \\
\hline Total & 520 & 100 \\
\hline
\end{tabular}

\section{Tabla IV \\ EDAD DE LAS MUJERES QUE USARON LA PÍLDORA ANTICONCEPTIVA DE EMERGENCIA}

\begin{tabular}{lccc}
\hline Mes & Usuarias $(\mathrm{n})$ & Promedio $\pm \mathrm{DE}$ & $\begin{array}{c}\text { Rango } \\
\text { de edad }\end{array}$ \\
\hline Enero 2012 & 16 & $25,8 \pm 9,5$ & $15-47$ \\
Febrero & 7 & $26,7 \pm 7,2$ & $17-38$ \\
Marzo & 31 & $25,1 \pm 7,6$ & $14-44$ \\
Abril & 38 & $23,9 \pm 6,8$ & $16-47$ \\
Mayo & 43 & $24,5 \pm 7,6$ & $15-50$ \\
Junio & 51 & $23,7 \pm 7,3$ & $13-49$ \\
Julio & 49 & $22,1 \pm 5,9$ & $16-46$ \\
Agosto & 23 & $21,8 \pm 7,3$ & $14-38$ \\
Septiembre & 33 & $21,0 \pm 4,8$ & $15-39$ \\
Octubre & 40 & $22,5 \pm 5,8$ & $15-40$ \\
Noviembre & 31 & $21,2 \pm 5,5$ & $15-37$ \\
Diciembre & 36 & $20,2 \pm 4,3$ & $14-32$ \\
Enero 2013 & 47 & $21,3 \pm 6,6$ & $14-45$ \\
Febrero & 19 & $25,3 \pm 8,9$ & $14-49$ \\
Marzo & 56 & $23,1 \pm 6,3$ & $15-41$ \\
\hline Total & 520 & $21,8 \pm 6,8$ & $13-50$ \\
\hline
\end{tabular}

Las razones principales para solicitar la PAE fueron el no uso de un método anticonceptivo $(49,8 \%)$ y la falla o uso incorrecto de éste $(49,6 \%)$. Sólo un caso fue por violación sexual (Tabla V). La mayoría de las mujeres que consultaron $(71,7 \%)$, no habían usado antes la PAE. El 21,1\% la había usado una vez y 2 veces el $2,3 \%$; solo 3 mujeres la habían usado entre 3 y 4 veces. Cuatro consultantes no sabían sobre el uso previo, dado que eran las parejas de las mujeres que requerían la PAE y que asistieron solos a la consulta. En 12 casos $(3,1 \%)$, no se registró esta información. El registro de este antecedente se inició a partir de junio de 2012 , por esta razón este dato fue analizado solo en 385 fichas clínicas y no en el total (Tabla VI).

\section{Tabla V \\ RAZONES PARA USAR LA PÍLDORA ANTICONCEPTIVA DE EMERGENCIA}

\begin{tabular}{lcc}
\hline Razón de uso & $\mathrm{n}$ & $\%$ \\
\hline No usó un anticonceptivo & 259 & 49,8 \\
Falló el anticonceptivo usado & 258 & 49,6 \\
Violación & 1 & 0,2 \\
Sin información & 2 & 0,4 \\
\hline Total & 520 & 100 \\
\hline
\end{tabular}

\section{Tabla VI \\ USO PREVIO DE LA PÍLDORA ANTICONCEPTIVA DE EMERGENCIA}

\begin{tabular}{lcc}
\hline $\begin{array}{l}\text { Veces que ha usado } \\
\text { PAE previamente* }\end{array}$ & $\mathrm{n}$ & $\%$ \\
\hline 0 & 276 & 71,7 \\
1 & 81 & 21,1 \\
2 & 9 & 2,3 \\
3 & 2 & 0,5 \\
4 & 1 & 0,3 \\
No sabe & 4 & 1,0 \\
Sin información & 12 & 3,1 \\
\hline Total & 385 & 100
\end{tabular}

* Este dato se empezó a registrar a partir de junio 2012. PAE: píldora anticonceptiva de emergencia

De las mujeres atendidas en Prosalud Chile, $46(8,8 \%)$ refirieron haber solicitado la PAE previamente en un centro público de salud donde se le negó su entrega por diversas razones. El rango de edad de estas usuarias fue de 13 a 35 años y el 
promedio de edad $21,8 \pm 5,6$ años. Las razones para solicitar la PAE fueron por no usar un anticonceptivo en 23 casos, por falla del condón masculino en 21 casos y el olvido de las píldoras anticonceptivas de rutina en 2 casos. Las razones reportadas por las usuarias por las cuales no se les entregó la PAE fueron diversas. Las más frecuentes fueron porque sólo se entregaba en casos de violación $(19,6 \%)$, no había hora de matrona $(19,6 \%)$ y no la entregaban en ninguna circunstancia $(15,2 \%)$ (Tabla VII). Los centros de salud que no entregaron la PAE pertenecen a diversas comunas de la Región Metropolitana y de otras regiones del país.

\section{DISCUSIÓN}

En Chile, se han realizado un gran número de investigaciones para determinar el mecanismo de acción de la PAE, pero hay escasos estudios sobre la demanda de la PAE en los servicios de salud, el perfil de las usuarias que usan este anticonceptivo, sus motivaciones y necesidades.

El número de consultas en las que se prescribió la PAE, representa el $25,6 \%$ del total de consultas de PAE. Estos datos son similares a los reportados por el Ministerio de Salud, en que el $26,4 \%$ del total de consultas de PAE requirieron la entrega de PAE (13). Esta situación se puede explicar porque no todas las mujeres y/o parejas que demandan la $\mathrm{PAE}$, requieren usarla. Al comparar el número de consultas y de prescripciones de PAE realizadas

\section{Tabla VII \\ RAZONES REPORTADAS POR LAS USUARIAS POR LAS CUALES EL ESTABLECIMIENTO PÚBLICO DE SALUD NO LES PROVEYÓ LA PÍLDORA ANTICONCEPTIVA DE EMERGENCIA}

\begin{tabular}{llc}
\hline Razones & $\mathrm{n}$ & $\%$ \\
\hline $\begin{array}{l}\text { La entregaban sólo en caso de } \\
\text { violación sexual }\end{array}$ & 9 & 19,6 \\
No había hora de matrona & 9 & 19,6 \\
No la entregaban & 7 & 15,2 \\
$\begin{array}{l}\text { No estaba inscrita en el centro de } \\
\text { salud }\end{array}$ & 5 & 10,9 \\
$\begin{array}{l}\text { No la entregaban por ser menor } \\
\text { de edad }\end{array}$ & 4 & 8,7 \\
$\begin{array}{l}\text { No tenían píldora anticonceptiva de } \\
\text { emergencia }\end{array}$ & 3 & 6,5 \\
Por la previsión de salud & 3 & 6,5 \\
Otras & 6 & 13,0 \\
\hline Total & 46 & 100 \\
\hline
\end{tabular}

en Prosalud Chile, con el número total de atenciones y recetas de PAE reportadas por el Ministerio de Salud en 2010 (13), se aprecia que las atenciones y prescripciones de PAE en Prosalud Chile representan aproximadamente el $45 \%$ de las realizadas en la red de establecimientos públicos de salud a nivel nacional. Esta cifra podría indicar que existe una demanda de PAE insatisfecha en la red pública de salud. Según estudios realizados por la Universidad Central en 2011 y 2013, 10,9\% y $5,9 \%$ de los municipios consultados telefónicamente, respectivamente, reportaron que no entregaban la PAE $(11,12)$. Nuestro estudio, encontró que el $8,8 \%$ de las mujeres que demandaron la PAE en consultorios municipalizados no la obtuvo por diversas razones.

La mayoría de las mujeres que solicitaron la PAE eran jóvenes, pero mayores de 15 años. Sólo el 1,5\% tenía menos de 15 años. El promedio de edad de las mujeres que usaron la PAE fue 21,8 \pm 6,8 años, cifra similar a la reportada en un estudio de usuarias de PAE de un consultorio público de salud de Santiago, que fue de 23,6 \pm 6,8 años (14). El rango etario también es similar en estos estudios, el 74,8\% tenía entre 15 a 26 años en nuestro estudio, y el 80,9\% tenía entre 15 a 23 años en el estudio citado anteriormente. Otro estudio, que analizó las variables socioeconómicas de las mujeres que demandaron la PAE en los servicios públicos de salud a nivel nacional en Chile, mostró que el 34,0\% de las mujeres tenía entre 15 a 19 años y el $66,0 \%$ tenía entre 20 a 44 años (13).

Las razones más frecuentes para solicitar la PAE son por haber tenido relaciones sexuales sin protección anticonceptiva o porque el método usado falló. Estas cifras son similares a las reportadas en dos estudios realizados en Chile, uno en un centro de salud público y el otro en uno privado. En el consultorio público, el $61,7 \%$ de las mujeres que solicitaron PAE, habían tenido un coito no protegido, el $24 \%$ le había fallado el anticonceptivo (rotura de condón y olvido de las pastillas) y el 4,3\% había sido víctima de un abuso sexual (14). En el centro de salud privado, $53 \%$ de las mujeres que solicitaron la PAE fue por no usar un anticonceptivo y $49 \%$ por falla del método (15). Algunas investigaciones realizadas en el mundo sobre el uso de la $\mathrm{PAE}$ en jóvenes, muestran resultados similares a este estudio $(16,17)$. En nuestro análisis, sólo una mujer solicitó la PAE después de una violación, lo que se puede explicar porque el centro no atendía consultas de emergencia, ni realizaba peritajes de delitos sexuales.

Uno de los argumentos de los grupos opositores al uso de la PAE, ha sido la preocupación de que este método se use frecuentemente, exponiendo a las mujeres a un mayor riesgo de embarazo no planeado e infecciones de transmisión sexual. Las investigaciones han mostrado que el uso repetido 
tiene una baja frecuencia. Los resultados de este estudio mostraron que el $92,8 \%$ de las mujeres no había usado nunca la PAE o la había usado una sola vez. Otro estudio, reportó que el $80,3 \%$ la había usado una o dos veces (18) y otro realizado en un servicio de urgencia reportó que de 446 mujeres que solicitaron la PAE, el $88 \%$ no la había usado antes (19).

Según las Encuestas Nacionales de Juventud $(20,21)$, el uso de la PAE en jóvenes de 15 a 29 años es bajo. Además, existen barreras en los servicios públicos de salud para proveerla $(11,12)$, pese a contar con una ley que asegura el acceso de las mujeres a la PAE sin discriminación, como un derecho garantizado por el Estado.

Para facilitar el acceso amplio y rápido a la $\mathrm{PAE}$, es necesario realizar estudios con usuarias y proveedores, para profundizar en los aspectos facilitadores y de barrera, que determinan su uso y prescripción.

\section{CONCLUSIONES}

La mayoría de las usuarias de la píldora de emergencia son jóvenes y la solicitan porque no usaron un anticonceptivo o éste les falló. El uso previo y frecuente es muy bajo. Aún existen barreras en el acceso en centros públicos de salud.

AGRADECIMIENTO: Los autores agradecen a las usuarias del estudio por su generosa colaboración. Esta investigación fue realizada gracias al apoyo financiero de Prosalud Interamericana.

\section{REFERENCIAS}

1. Organización Mundial de la Salud. Planificación familiar. Un manual mundial para proveedores. Actualización modificada en 2011. Disponible en: http://www. fphandbook.org/sites/default/files/hb_spanish_2012. pdf

2. Organización Mundial de la Salud. Hoja informativa sobre la seguridad de las Píldoras Anticonceptivas de Emergencia de Levonorgestrel solo (PAELNG). 2010. Disponible en: http://whqlibdoc.who.int/ hq/2010/WHO_RHR_HRP_10.06_spa.pdf

3. Díaz S, Croxatto HB. Anticoncepción de emergencia. En: Ginecología. 3 ${ }^{a}$ Edición. Pérez Sánchez A \& Donoso Siña E (eds). Editorial Mediterráneo, Santiago, Chile, 2003. pp. 1067-73.

4. Raymond E, Trussell J, Polis C. Population effect of increased access to emergency contraceptive pills. A systematic review. Obstet Gynecol 2007;109:181-82.

5. Westley E, von hertzen H, Faúndes A. Expanding access to emergency contraception. Int J Gynecol Obstet 2007;97:235-37.

6. Schiappacasse V. Acceso a anticoncepción de emergencia como derecho de la mujer. In: Direitos sexuais e reprodutivos das mulheres: A contribuição da pesquisa em ciências sociais. Osis MJD; Bahamondes L (Org.). Campinas: Cemicamp, 2013:101-21.

7. Ministerio de Salud. Gobierno de Chile. Normas y guía clínica para la atención en servicios de urgencia de personas víctimas de violencia sexual. 2004. Disponible en: http://www.scielo.cl/pdf/rchog/v70n1/ art11.pdf

8. Ministerio de Salud, Instituto Chileno de Medicina Reproductiva y Asociación Chilena de Protección de la Familia. Normas Nacionales sobre Regulación de la Fertilidad.Chile, Septiembre, 2006. Disponible en: http://web.minsal.cl/portal/url/item/795c63caff4ede9fe 04001011f014bf2.pdf

9. Ministerio de Salud. Decreto 194. Aprueba Formulario Nacional de Medicamentos. 2005. Disponible en: http://www.leychile.cl/Navegar?idNorma=247938\&id Parte $=0$.

10. Schiappacasse V, Díaz S. Chile: "One step forward, one step back". In: Emergency Contraception. The Story of a Global Reproductive Health Technology. Chapter 7. (eds) Foster AM, Wynn LL. Palgrave Macmillan, New York, N.Y., U.S.A., 2012. pp. 107-22.

11. Dides C, Benavente C, Sáez I, Nicholls L, Correa T. Provisión de anticoncepción de emergencia en el Sistema de Salud Municipal de Chile. Estado de Situación 2011. Disponible en: http://www.ucentral. cl/prontus_ucentral2012/site/artic/20120711/asocfile/20120711091025/provision_de_ae_2011.pdf

12. Dides D, Nicholls L, Bozo N, Fernández C. Provisión de Anticoncepción de Emergencia en el Sistema de Salud Municipal de Chile. Estado de Situación 2013. Disponible en: http://banhodeassento.files.wordpress. com/2014/01/estado-situacic3b3n-entrega-ae-2013cesop-facso.pdf

13. Morán JM. La anticoncepción de emergencia en Chile: estructuración de su demanda en función de variables socioeconómicas. Rev Bras Estud Popul 2013;30(1):125-44.

14. Escobar B, Román C, Muñoz C, López F. Perfil biosocial de las usuarias que demandan la píldora de anticoncepción de emergencia en un centro de salud de Santiago, 2006-2007. Rev Chil Obstet Ginecol 2008;73(4):223-7.

15. Noé G, Croxatto HB, Salvatierra AM, Reyes V, Villarroel C, Muñoz C, et al. Contraceptive efficacy of emergency contraception with levonorgestrel given before or after ovulation. Contraception 2011;84(5):486-92.

16. Antona Rodríguez A, Madrid Gutiérrez J. Anticoncepción de emergencia, adolescencia y representaciones sociales. Sexología Integral 2009;6(3)102-8.

17. Pearson VA, Owen MR, Phillips DR, Pereira Gray DJ, Marshall MN. Pregnant teenagers' knowledge and use of emergency contraception. BMJ 1995;310:1644.

18. Ros C, Miret M, Rué M. Estudio descriptivo sobre el uso de la anticoncepción de emergencia en Cataluña. Comparación entre una zona rural y una urbana. Gac Sanit 2009;23(6):496-500. 
19. Cárdenas Cruz DP, Parrilla Ruiz F, Mengíbar Gómez MM, Espinosa Fernández M, Issa Khozouz Z, Cárdenas Cruz A. Perfil de la solicitante de la píldora postcoital (Levonorgestrel) en unidades de urgencias. Medicina de Familia (And) 2002;3(3):179-83.

20. Instituto Nacional de la Juventud. Sexta Encuesta Nacional de Juventud 2009. Santiago de Chile: Ministerio de Desarrollo Social, Gobierno de Chile;
2010. Disponible en: http://www.injuv.gob.cl/portal/ wp-content/files_mf/sextaencuestanacionaldejuventud.pdf.

21. Instituto Nacional de la Juventud. Séptima Encuesta Nacional de Juventud 2012. Santiago de Chile: Ministerio de Desarrollo Social, Gobierno de Chile; 2012. Disponible en: http://www.injuv.gob.cl/portal/wp-content/files_mf/septimaencuestanacionaljuventud2.pdf. 